OPEN@ACCESS

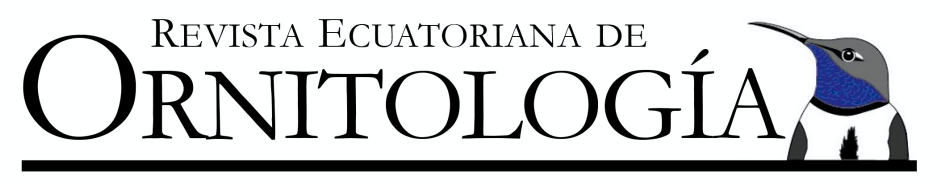

NOTAS DE CAMPO/FIELD NOTES

\title{
Tucanete Lomirrojo Aulacorhynchus haematopygus depredando a un colibrí
}

\author{
Hans Heinz \\ Pajareando Ando Ecuador,e-mail: hansheinz67@yahoo.com \\ Editado por/Edited by: Héctor Cadena-Ortiz \\ Recibido/Received: 09 Abril 2020. Aceptado/Accepted: 18 Octubre 2020 \\ Publicado en línea/Published online: 04 Diciembre 2020
}

Usualmente, los tucanes (Ramphastidae) son considerados frugívoros. Sin embargo, existen reportes de otros ítems alimenticios en su dieta, incluyendo insectos grandes, vertebrados pequeños, huevos y pichones de otras aves (Remsen et al., 1993).

El 22 de abril de 2017, alrededor de las 9h00, fotografié un individuo de Tucanete Lomirrojo Aulacorhynchus haematopygus en Tandayapa Bird Lodge $(0,000069,-78,677751,1740$ m s.n.m.), provincia de Pichincha, depredando a una Coronita Colianteada Boissonneaua flavescens (Fig. 1). Este tucanete saltaba en las ramas alrededor de los bebederos para colibríes, y de manera rápida se lanzó y atrapó con el pico al colibrí en vuelo. Este luchó por liberarse, sin éxito. El tucanete se perchó en una rama cercana a c. 1,5 m del suelo, donde compactó a su presa usando patas y pico, y la engulló entera. Todo el evento duró menos de un minuto.

Remsen et al. (1993) encontraron restos de artrópodos, huevos y vertebrados en únicamente 6,7\% de 326 contenidos estomacales de 32 especies de tucanes. Por su amplio muestreo, sugieren que la no frugivoría en tucanes está sobredimensionada. No obstante, otros autores sugieren que los tucanes son más oportunistas de lo esperado con base en varios eventos de depredación de nidos por diferentes especies de tucanes (Menezes \& Marini, 2017; Cove et al., 2017). Incluso se ha reportado la depredación de A. haematopygus a una nidada de tres huevos de Tinamú Grande Tinamus major (Arias-Alzate et al., 2012), así como la depredación de varios huevos de Perico de El Oro Pyrrhura orcesi (Garzón-Santomaro et al., 2020). Con todo, este parece ser el primer reporte de un tucán depredando un ave en vuelo.

Agradezco a Héctor Cadena por la motivación para escribir este manuscrito y a los dos revisores anónimos por sus sugerencias y comentarios.

\section{REFERENCIAS}

Arias-Alzate, A., Delgado-V, C.A., Botero-Cañola, S., \& Sánchez-Londoño, J.D. (2012). Un evento de depredación del tucancito rabirojo, Aulacorhynchus haematopygus (Piciformes: Ramphastidae), sobre huevos de tinamú grande Tinamus major (Tinamiformes: Tinamidae). Brenesia, 78, 88-89.

Cove, M.V., Fernández, C.M., Álvarez, M.V., Bird, S., Jones, D.W., \& Fagan, M.E. (2017). Toucans descend to the forest floor to consume the eggs of ground-nesting birds. Food Webs, 10, 2-4. DOI: http://dx.doi.org/10.1016/j.fooweb.2017.01.003

Garzón-Santomaro C., Naranjo-Saltos, E., \& Pozo-Zamora, G. (2020). Depredación de nidos del perico de El Oro Pyrrhura orcesi por el tucanete lomirrojo Aulacorhynchus haematopygus, en la Reserva Buenaventura, Ecuador. Huitzil, 21(1), e-532. DOI: https://doi.org/10.28947/hrmo.2020.21.1.397

Menezes, J.C., \& Marini, M.Â. (2017). Predators of bird nests in the Neotropics: a review. Journal of Field Ornithology, 88(2), 99-114. DOI: https://doi.org/10.1111/jofo.12203 
Remsen Jr, J.V., Hyde, M.A., \& Chapman, A. (1993). The diets of Neotropical trogons, motmots, barbets and toucans. The Condor, 95(1), 178-192. DOI: https://doi.org/10.2307/1369399

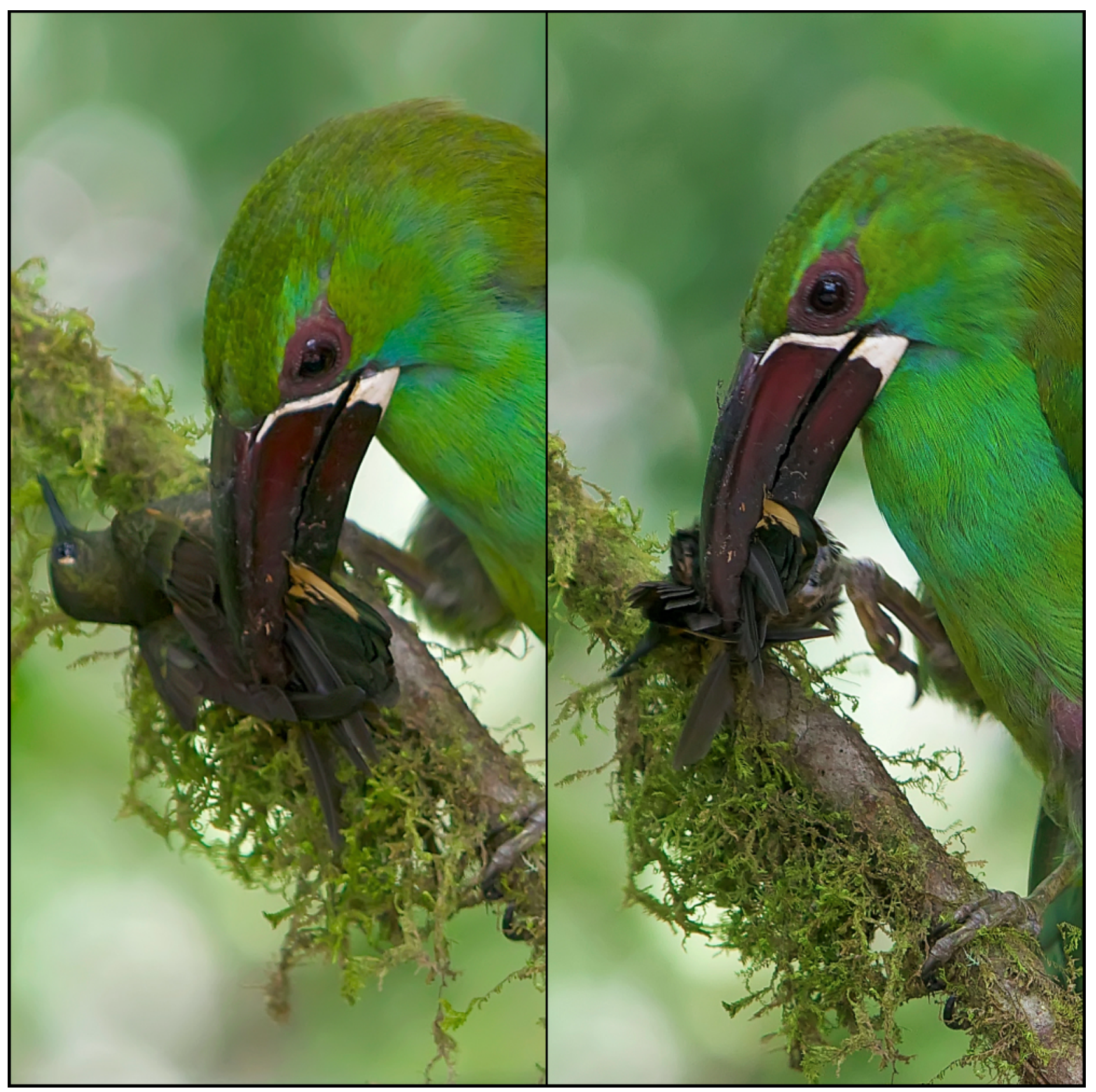

Figura 1: Tucanete Lomirrojo Aulacorhynchus haematopygus depredando a una Coronita Colianteada Boissonneaua flavescens, 22 de abril de 2017, Tandayapa Bird Lodge, Pichincha, Ecuador (Hans Heinz). 